If we make this assumption it follows that $\mathfrak{M}$ annuls $\partial A / \partial y_{1 r}$, where $r$ is the order of $A$ in $y_{1}$. Let $s$ be the order of $A$ in $y_{2}$. We form the resultant $R$ of $A$ and $\partial A / \partial y_{1 r}$, considered as algebraic polynomials in $y_{28}$. Since $A$ is irreducible, and cannot be a factor of $\partial A / \partial y_{1 r}$, $R$ is a nonzero polynomial, free of $y_{2 s}$, which is annulled by $\mathfrak{M}$. Since $R$ is of lower efiective order than $A$ in $y_{2}, \mathfrak{M}$ must be an essential singular manifold of $A$ relative to $y_{2}$. The proof is now complete.

RUTGERS UNIVERSITY

\title{
DISTINCT REPRESENTATIVES OF SUBSETS
}

MARSHALL HALL, JR.

1. Introduction. Let $W$ be a set of elements $a_{i}^{\prime} \cdot W=\left\{a_{1}, \cdots\right\}$ and let $U\left\{S_{1}, \cdots, S_{j}, \cdots\right\}$ be an indexed system of subsets of $W$. We wish to choose distinct representatives of the subsets. If $a_{j}=R\left(S_{j}\right)$ designates the representative of the subset $S_{j}$, then we require $R\left(S_{j}\right) \in S_{j}$ for all $j$ and $R\left(S_{j}\right) \neq R\left(S_{k}\right)$ if $j \neq k$. It is to be emphasized that subsets are distinguished only by their indices and distinct subsets may contain the same elements. An obviously necessary condition for the existence of distinct representatives is:

Condition C: Every $k$ distinct subsets contain between them at least $k$ distinct elements, for every finite $k$. P. Hall ${ }^{1}$ has shown that if the number of subsets is finite, condition $C$ is also sufficient for the existence of a system of distinct representatives, or SDR as we shall abbreviate. This condition is no longer sufficient if the number of subsets is infinite. As a counter example consider $U\left(S_{0}, S_{1}, \cdots\right\}$ where $S_{0}=\left\{a_{1}, a_{2}, \cdots\right\}, S_{i}=\left\{a_{i}\right\}, i=1,2, \cdots$. Here condition C is easily shown to hold for the subsets, but clearly no representative may be selected for $S_{0}$ which is not also a representative of some $S_{i}$.

In this paper it is shown that condition $C$ is sufficient if every subset $S_{j}$ is finite, and also an estimate on the number of systems of distinct representatives is given. This latter result is applied to Latin squares.

Theorem 1. Given an indexed system $U\left\{S_{1}, \cdots, S_{j}, \cdots\right\}$ of finite subsets of a set $W\left\{a_{1}, \cdots, a_{i}, \cdots\right\}$. If the subsets satisfy condi1947.

Received by the editors October 21,1947 , and, in revised form, November 8 ,

${ }^{1}$ P. Hall, On representatives of subsets, J. London Math. Soc. vol. 10 (1935) pp. 2630 . 
tion $\mathrm{C}$ for every finite $k$ then there exists a system of distinct representatives $R\left(S_{j}\right)$ of the subsets.

THEOREM 2. Under the hypotheses of Theorem 1 if the smallest subset contains $r$ distinct elements, then there are at least $r$ ! different systems of distinct representatives.

TheOREM 3. There are at least $n !(n-1) ! \cdots 2 ! \cdot 1$ ! different $n$ by $n$ Latin squares.

2. Blocks and critical blocks. A finite system of subsets $\left\{S_{j}\right\}$ of $U\left\{S_{1}, \cdots, S_{j}\right\}$ together with the set of elements $\left\{a_{i}\right\}$ of these subsets will be called a block $B$. If we wish to indicate that there are $r$ subsets and $s$ distinct elements in the block $B$, we write $B=B_{r, s}$. We shall assume that every subset $S_{j}$ contains only a finite number of elements. Condition $\mathrm{C}$ is equivalent to the condition $s \geqq r$ for every block $B_{r, s}$ and we shall assume this to hold. If $s=r$ we say the block $B_{r, r}$ is a critical block.

There is a natural partial ordering for the blocks, if we define $B \subseteq B^{\prime}$ whenever every subset $S_{j}$ of the block $B$ is a subset of the block $B^{\prime}$. With respect to this partial ordering it is easily verified that the blocks form a distributive lattice, since they are essentially finite sets of $S_{j}$ 's. It will be desirable to consider the void block as an improper critical block $B_{0,0}$.

LEMMA 1. The union $B_{k, k} \cup B_{l, l}$ and crosscut $B_{k, k} \cap B_{l, l}$ of critical blocks are again critical blocks.

Proof. Given two critical blocks $B_{k, k}$ and $B_{l, l}$, let $B_{k, k} \cap B_{l, l}=B_{r, s}$ and $B_{k, k} \cup B_{l, l}=B_{u, v}$. Let $S_{1}, \cdots, S_{r}$ be the subsets and $a_{1}, \cdots, a_{s}$ be the elements of $B_{r, s}$. Then $s \geqq r$. In $B_{k, k} \cup B_{l, l}=B_{u, v}$, we have $u=k+l-r$ since there are in $B_{u, v}$ the sets $S_{1}, \cdots, S_{r}$ and $k-r$ further sets of $B_{k, k}$ and $l-r$ further sets of $B_{l, l}$. Also in $B_{u, v}$ there are at most $k+l-s$ different elements since at least the elements $a_{1}, \cdots, a_{s}$ appear in both $B_{k, k}$ and $B_{l, l}$. Hence $k+l-s \geqq v \geqq u$ $=k+l-r$ and also $s \geqq r$, whence $s=r, v=u$ and both $B_{r, s}$ and $B_{u, v}$ are critical blocks.

LEMMA 2. Given an indexed system of finite subsets $U\left\{S_{1}, \cdots\right.$, $\left.S_{j}, \cdots\right\}$ of a set of elements $W\left\{a_{1}, \cdots, a_{i}, \cdots\right\}$ satisfying condition $\mathrm{C}$ and a proper critical block $B_{k, k}$ of $U$. If the system $U^{\prime}\left\{S_{1}^{\prime}, \cdots\right.$, $\left.S_{j}^{\prime}, \cdots\right\}$ consists of the subsets $S_{i}^{\prime}=S_{i}$ for $S_{i} \in B_{k, k}$, and, for $S_{j}$ $\notin B_{k, k}, S_{j}^{\prime}$ contains those elements of $S_{j}$ which are not elements of $B_{k, k}$, then $U^{\prime}\left\{S_{1}^{\prime}, \cdots, S_{j}^{\prime}, \cdots\right\}$ also satisfies condition C.

Proof. This lemma can be more loosely phrased by saying that 
deletion of elements of a critical block $B_{k, k}$ from sets not in $B_{k, k}$ does not alter the validity of condition C. Let $B_{r, s}$ be any block of $U$ and $B_{r, t}^{\prime}$ the corresponding block of $U^{\prime}$ (no subsets have been deleted). Also let $B_{r, s} \cap B_{k, k}=B_{l, f}, B_{r, s} \cup B_{k, k}=B_{m, n}$. The number of elements of $B_{r, s}$ not in $B_{k, k}$ is $n-k$. As $n \geqq m=r+k-l$, we have $n-k \geqq r-l$. In the deleted block $B_{r, t}$ there are $l$ subsets of $B_{k, k}$ and $r-l$ other subsets and there are $f \geqq l$ elements of $B_{k, k}$ and $n-k$ other elements. Hence $t=f+n-k \geqq l+r-l=r$ and so condition $\mathrm{C}$ is also satisfied for the deleted block $B_{r, t}^{\prime}$, and hence for the deleted system $U^{\prime}$.

Since an element of a critical block could not be a representative of any subset not in that block, the deleted system $U^{\prime}$ will have the same SDR's (if there are any) as the system $U$.

\section{The principal theorems.}

Proof of Theorem 1. We divide the proof into two cases, according to the cardinal number $\alpha$ of sets $S_{j}$ in $U$ : Case 1: $\alpha$ finite, Case 2: $\alpha$ infinite.

Case 1: $\alpha$ finite. Suppose first there is a critical block $B_{k, k}, 1 \leqq k$ $\leqq \alpha-1$, not the whole system $U$. From the sets of $U$ not in $B_{k, k}$ delete the elements of $B_{k, k}$. Then $U^{\prime}$, the deleted system, consists of $B_{k, k}$ and another block $B_{\alpha-k, 0}^{\prime}$ with no sets or elements in common. By Lemma 2, $U^{\prime}$ satisfies condition $\mathrm{C}$, whence both $B_{k, k}$ and $B_{\alpha-k, 0}^{\prime}$ as systems $U^{\prime}$ and $U^{\prime \prime}$ satisfy condition $\mathrm{C}$. By induction both of these have SDR's and, as they are disjoint, together these SDR's form an SDR for $U$. Now suppose there is no critical block except possibly the whole system $U$. This means that for an arbitrary block $B_{r, s}$ with $r \leqq \alpha-1$ we have $s \geqq r+1$. Now take an arbitrary element of $S_{\alpha}$ as a representative and delete this element in every other subset. In this deletion any block $B_{r, s}$ in $S_{1}, \cdots, S_{\alpha-1}$ goes into a block $B_{r, t}^{\prime}$ where $t=s$ or $s-1$ since only one element has been deleted. As $s \geqq r+1$, $t \geqq r$ and so Condition $\mathrm{C}$ holds for $U^{\prime}\left(S_{1}^{\prime}, \cdots, S_{\alpha-1}^{\prime}\right)$ whence by induction there is an SDR for $U^{\prime}$ and this together with the representative for $S_{\alpha}$ gives an SDR for $U$.

This part of the theorem is equivalent to Philip Hall's result.

Case 2: $\alpha$ infinite. If from every set $S_{j}$ of $U$ we delete an element $a_{1}$ which does not belong to any critical block, then the deleted system still satisfies condition C. For in any block $B_{r, s}$ which contains $a_{1}$ we have $s \geqq r+1$, and after deletion the block becomes $B_{r, s-1}^{\prime}$ with $s-1 \geqq r$. Condition $\mathrm{C}$ is a property of finite character in the sense used by Tukey2 since it applies to finite collections of subsets $S_{j}$. If

${ }^{2} \mathrm{~J}$. W. Tukey, Convergence and uniformity in topology, Annals of Mathematics Studies, No. 2, 1940, p. 7. 
we consider the set $T$ of pairs $\left(a_{i}, S_{j}\right), a_{i} \in S_{j}$, the following property of subsets $T^{*}$ of $T$ will also be a property of finite character. Property D: "After deleting $a_{i}$ from $S_{j}$ for every pair $\left(a_{i}, S_{j}\right) \in T$, the system $U\left\{S_{j}\right\}$ still satisfies Condition C." Hence, using the third form of Zorn's Lemma ${ }^{3}$ Tukey $^{2}$ p. 7), we may conclude that there is a maximal system $T^{*}$ with property $\mathrm{D}$. Put more simply, there is a maximal amount of deleting of elements $a_{i}$ from subsets $S_{j}$ which may be done without destroying the validity of Condition $C$. Let us suppose this done. Then by the first sentence of this paragraph, every element belongs to a critical block. From Lemma 2, every critical block is disjoint from all sets not in it. Hence we have left only disjoint critical blocks. By Case 1, a critical block, being finite, possesses an SDR. Hence within each critical block deletion may continue until each $S_{j}$ contains only one element. Hence after maximal deletion there is exactly one element left in each set, and as Condition $\mathrm{C}$ is still satisfied, we have left an SDR for $U$.

The reader will note that if initially every element of $U$ belongs to a critical block, the argument goes through without appeal to the axiom of choice.

Proof of THEOREM 2. Two systems of distinct representatives are different if they give different representatives for any subset. This theorem gives a lower limit on the number of different SDR's. I assert that in $U$ there is one $S_{j}$ in which an aribtrary element may be taken as a representative in an SDR. Suppose $U$ contains critical blocks. Choose a minimal critical block $B_{k, k}$. Here an $S_{j}$ in $B_{k, k}$ has the property that any element of $S_{j}$ may be a representative. For deleting a chosen element of $S_{j}$ from the other subsets of $B_{k, k}$ leaves them satisfying condition $C$, whence the chosen element appears in an SDR for $B_{k, k}$. But in any SDR for $U$ we may replace any SDR for $B_{k, k}$ by any other. On the other hand, if $U$ contains no critical blocks, we may use an arbitrary representative from any one subset $S_{j}$ without violating condition $\mathrm{C}$ by deleting it from the remaining subsets. Hence given $U$ with at least $r$ elements in every $S$, we may choose fron an appropriate $S_{j}$ a representative in at least $r$ ways and delete it elsewhere without violating condition C. Now apart from this $S_{j}$ every other deleted subset contains at least $r-1$ elements. By induction this possesses at least $(r-1)$ ! different SDR's. Hence $U$ possesses at least $r(r-1) !=r$ ! different SDR's.

4. Application to Latin squares. Theorem 2 may be used to improve

${ }^{3}$ Cf. C. Kuratowski, L'elimination des nombres transfinis, Fund. Math. (1922) pp. 76-108, Theorem 42, p. 89. 
a result of the author's ${ }^{4}$ on Latin squares. Given a Latin rectangle with $n$ columns and $r<n$ rows. The problem of finding an $(r+1)$ st row to add to this Latin rectangle is equivalent to finding an SDR for the $n$ sets $C_{1} \cdots C_{n}$ where each $C_{i}$ consists of the $n-r$ letters not in the ith column. Applying theorem 2, we see that this may be done in at least $(n-1)$ ! ways. Hence adding a row at a time we see that the number of distinct $n$ by $n$ Latin squares is at least $n !(n-1) ! \cdots 2$ ! $1 !$.

This number is surely too small. A better estimate may be obtained by combining the results given here with those of Erdös and Kaplansky. ${ }^{5}$ This would increase the first $k$ factors for $k<(\log n)^{3 / 2-e}$. The last factors are also too small and it would be interesting to obtain further improvements.

Note added in proof. Another proof of Theorem 1, by C. J. Everett and George Whaples, is to appear in Amer. J. Math. See Bull. Amer. Math. Soc. Abstract 53-5-170.

\section{Ohio State University}

4 Marshall Hall, An existence theorem for Latin squares, Bull. Amer. Math. Soc. vol. 51 (1945) pp. 387-388.

${ }^{5} \mathrm{P}$. Erdös and I. Kaplansky, The asymptotic number of Latin rectangles, Amer. J. Math. vol. 68 (1946) pp. 230-236. 